\title{
RECOVERY OF HAND GRASPING BY TRANSPOSITION OF THE FINGERS, FINGER STUMPS, AND METACARPAL BONES AFTER INJURIES AND THEIR CONSEQUENCES
}

\author{
Alexandrov N.M., Kiselev D.V., Petrov S.V. \\ Federal State-Financed Educational Institution of Higher Education «Research Medical University \\ of Volga Region of the Russian Ministry of Health», Nizhny Novgorod, e-mail: info@ nniito.ru
}

Aims.

To improve the efficacy of the finger repair method due to transposition of hand segments, including cicatricial and deformed ones.

Materials and methods.

The article presents the experience of surgical treatment of 184 patients with traumatic absence of fingers through the use of donor resources of the damaged hand itself. The fingers were repaired by transposition of the intact, defective finger, finger stump, or metacarpal bone. Thumb repair was carried out in 177 cases, pointer finger - in 12, middle finger - in 5, ring finger - in 8, and little finger - in 1 case. Recovery of one finger (usually the thumb) was implemented in 186 cases, the thumb and one of the triphalangeal fingers - in 5 cases, the thumb and two triphalangeal fingers - in 1 case, and two triphalangeal fingers - in 2 cases. With tissues that were intact and not very altered, the prevention of ischemic complications was performed by preserving or repairing the finger vessels and veins. With pathologically altered tissues, the segment was transpositioned in two stages, after its presurgical training. If there was a short finger donor stump, distraction of pedicles was additionally carried out.

Results.

The developed methods based on ischemic preconditioning, preforming of the transpositioned segments, and distraction of pedicles, extended the potential of using the pathologically changed tissues, provided sturdy engraftment and recovery of the hand grasping with minimal donor deformity. The immediate and long-term treatment outcomes were analysed. The method for mechanical training of the donor finger for ischemia before its transposition was suggested. This method has provided the possibility for transposition of any hand segment regardless of its location relative to the finger under repair, scarring severity, and nature of the hand deformation. The possibility for transposition of the finger stumps and metacarpal bones with more proximal amputation levels $(P=0.01)$ and ulnar location $(P=0.0001)$ was shown.

Conclusions.

Application of the method allowed for recovery of hand grasping in $93.3 \%$ of the victims, despite the heavy scar deformation of the donor segment and the hand caused by mechanical, gunshot, thermal, and combined injuries.

Keywords: amputation, pollicisation, transposition of finger stumps and metacarpal bones, ischemic preconditioning of the tissues, delay phenomenon, preforming, operative technique.

INTRODUCTION. Transposition of fingers, finger stumps, and metacarpal bones is not widespread for a number of reasons [1]. A significant limiting factor is a potential risk of the development of ischemic complications when cicatricial and deformed segments are used as donor parts. Vascular complications lead to partial or complete necrosis of the skin flaps and the transferred segment in $11 \%$ of cases even in patients with barely altered or intact tissues [2-4]. The effectiveness of the methods of prevention of such complications is often low. Thus, cicatricial tissues are not transferred. Such reconstructive operations are performed only in patients with some types of hand deformities, the presence of digital arteries, and certain conditions of soft tissues and bones of the donor segment $[1 ; 5]$. Specialists prefer a more damaging but more technically convenient method of transposition of finger II or the finger adjoining to the one to be reconstructed 
or its stump. This provides a reduction of the donor part damage and the degree of blood circulation disturbances in the transferred segment due to a milder injury of vascular bundles and the transferred skin flaps $[1 ; 5]$. However, the existing technologies do not allow surgeons to transpose short finger and metacarpal stumps located far from the recipient area and deformed fingers in patients with damaged great vessels and severe disturbances of tissue blood supply [5]. The specified factors prevent the vast application of this method that could open new perspectives of the use of the tissue and functional reserve of the injured hand for the reconstruction of a finger, minimization of donor area damage, and improvement of the operation outcome. Thus, there is a necessity in the investigating opportunities for the expansion of indications for the transposition of pathologically altered segments of the hand and prevention of ischemic complications in the reconstructed finger.

The aim of the study was to expand a set of indications for the method of reconstruction of fingers with transposition of hand segments, including cicatricial and deformed ones.

\section{MATERIALS AND METHODS.}

The study protocol followed guidelines for experimental investigation with human subjects in accordance with the Declaration of Helsinki and was approved by the ethics committee. Written informed consent was obtained from each patient (or official representative) before the study.

The authors analyzed the results of the treatment of 184 patients with 189 hands that underwent primary and secondary reconstruction of 203 fingers by the method of transposition of different segments of the deformed hand with conventional (85 fingers) and original (118 fingers) techniques. In the majority of cases, mechanical injuries of hand were observed (101 - 54.9\%). Besides, there were gunshot wounds $(23-12.5 \%)$, consequences of burns $(27-14.7 \%)$, freezing injuries $(30-16.3 \%)$, and combined injuries $(3-1.6 \%)$. The right hand was injured in 80 patients (43.5\%), the left hand - in 68 patients $(37.0 \%)$, bilateral finger and hand stumps - in 36 patients (19.6\%). There were 161 male patients $(87.5 \%)$ and 23 female patients (12.5\%). Home accidents were registered in 100 patients $(54.3 \%)$, industrial injuries - in 79 patients (42.9\%), and injuries during military service - in 5 patients $(2.7 \%)$. The average age of patients was $28.18 \pm 3.74$ years old.

The analysis of the clinical data allowed the authors to identify the following types of hand deformities (Figure 1). 


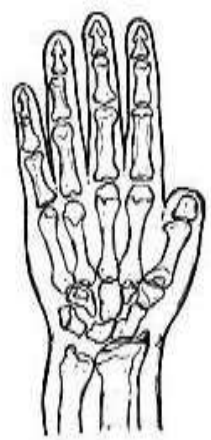

Type I $(58-30.8 \%)$

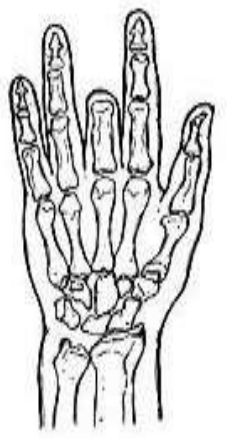

Type VI $(1-0.5 \%)$

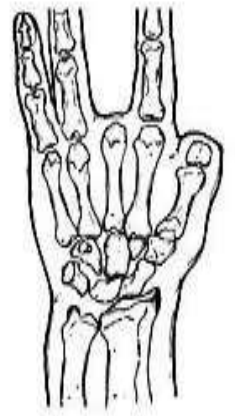

Type II $(36-19.0 \%)$

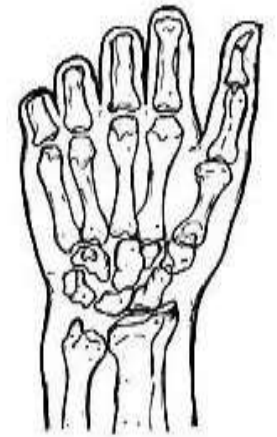

Type VII $(3-1.6 \%)$

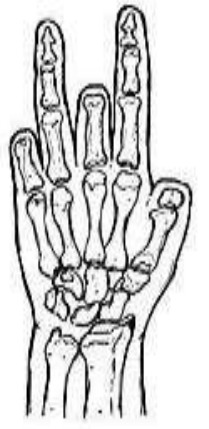

Type III $(35-18.5 \%) \quad$ Type IV $(1-0.5 \%)$
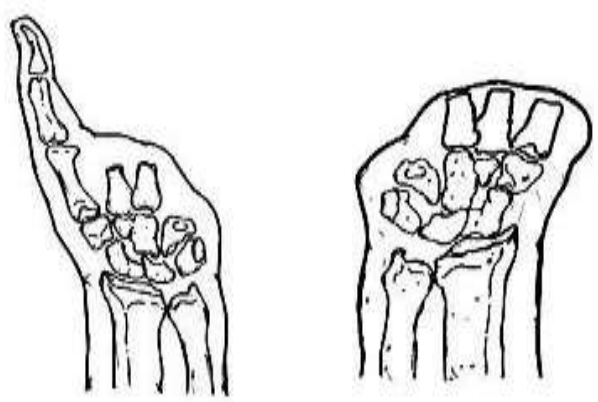

Type VIII $(1-0.5 \%)$

Type IX (7 - 3.7\%)

Figure 1. Types of hand deformities

The character of the transferred segments in patients with different types of deformities and types of reconstruction is presented in Table 1. Thumb reconstruction was performed in 177 of cases, reconstruction of finger II - in 12 cases, reconstruction of finger III - in 5 cases, reconstruction of finger IV - in 8 cases, and reconstruction of finger V - in 1 case. Reconstruction of one finger (primarily, finger I) was performed in 186 cases, I and one of three-phalange fingers in 5 cases, I and two of three-phalanges fingers - in 1 case, and two three-phalanges fingers - in 2 cases.

Table 1. The character of the transferred segments in patients with different types of hand deformities and types of reconstruction

\begin{tabular}{|l|l|l|l|l|l|l|l|l|l|l|l|}
\hline \multirow{2}{*}{ Transposition of segments } & \multicolumn{7}{|c|}{ Types of hand deformities } & \multicolumn{2}{l|}{ Total (hands) } \\
\cline { 2 - 11 } & & I & II & III & IV & V & VI & VII & VIII & IX & \\
\hline Primary reconstruction: & & & & & & & & & & & \\
\hline intact finger & 8 & & & & & & & & & 8 \\
\hline
\end{tabular}




\begin{tabular}{|l|l|l|l|l|l|l|l|l|l|l|}
\hline deformed finger & 3 & 2 & 1 & & & 1 & & & & 7 \\
\hline $\begin{array}{l}\text { transposition of a segment after flap } \\
\text { surgery }\end{array}$ & 3 & 1 & & & & & & & & 4 \\
\hline Secondary reconstruction: & 18 & & & & & & & & & 18 \\
\hline intact finger (typical) & 22 & 5 & & & & & & & & 27 \\
\hline deformed finger (typical) & & 13 & 12 & 1 & 14 & & & & & 40 \\
\hline finger stump (typical) & & 2 & 4 & & 11 & & & & 1 & 18 \\
\hline metacarpal bone stump (typical) & 3 & & & & & & & & & 3 \\
\hline distracted finger & & 9 & 12 & & 8 & & & & & 29 \\
\hline distracted finger stump* & 1 & 4 & 6 & & 14 & & 3 & 1 & 6 & 35 \\
\hline distracted metacarpal bone stump* & 58 & 36 & 35 & 1 & 47 & 1 & 3 & 1 & 7 & 189 \\
\hline Total (hands): & & & & & & & & \\
\hline
\end{tabular}

*One or more stumps or their parts were transferred within one hand

The levels of amputation of the reconstructed finger are presented in Table 2. It was also shown that the transposition of finger and metacarpal bone stumps after distraction was primarily used in patients with distal amputations. As a rule, the stump of finger II or the adjoining finger was transferred, and after the distraction, ulnar stumps at proximal levels.

Table 2. The character of the transferred segments at different levels of stumps of the reconstructed fingers

\begin{tabular}{|c|c|c|c|c|c|c|c|c|c|}
\hline $\begin{array}{l}\text { Levels of amputation of the } \\
\text { reconstructed finger }\end{array}$ & 1 & 2 & 3 & 4 & 5 & 6 & 7 & 8 & Total (fingers) \\
\hline $\begin{array}{l}\text { Methods of finger } \\
\text { reconstruction }\end{array}$ & & & & & & & & & \\
\hline Primary reconstruction: & & & & & & & & & \\
\hline $\begin{array}{l}\text { transposition of the intact } \\
\text { finger }\end{array}$ & & & & 3 & 5 & & & & 8 \\
\hline $\begin{array}{l}\text { transposition of the distal part } \\
\text { of the deformed finger }\end{array}$ & & & 1 & & 2 & 1 & 1 & & 5 \\
\hline
\end{tabular}




\begin{tabular}{|c|c|c|c|c|c|c|c|c|c|}
\hline $\begin{array}{l}\text { transposition of the finger } \\
\text { stump }\end{array}$ & & 1 & & & 1 & & & & 2 \\
\hline $\begin{array}{l}\text { transposition of the segment } \\
\text { after flap surgery }\end{array}$ & & & & & 1 & 1 & & 2 & 4 \\
\hline Secondary reconstruction: & & & & & & & & & \\
\hline $\begin{array}{l}\text { transposition of the intact } \\
\text { finger }\end{array}$ & & & & 4 & 10 & 3 & & 1 & 18 \\
\hline $\begin{array}{l}\text { transposition of the deformed } \\
\text { finger }\end{array}$ & 1 & & 1 & 8 & 7 & 1 & 2 & 7 & 27 \\
\hline $\begin{array}{l}\text { transposition of the finger } \\
\text { stump (typical) }\end{array}$ & & 1 & 1 & 18 & 15 & 2 & 1 & 3 & 41 \\
\hline $\begin{array}{l}\text { transposition of the metacarpal } \\
\text { bone stump (typical) }\end{array}$ & & & & 3 & 7 & 4 & 1 & 2 & 17 \\
\hline $\begin{array}{l}\text { transposition of the finger after } \\
\text { distraction }\end{array}$ & & & & 1 & & 1 & & 1 & 3 \\
\hline $\begin{array}{l}\text { transposition of the finger } \\
\text { stump after distraction }\end{array}$ & & & 1 & 14 & 13 & 4 & 1 & & 33 \\
\hline $\begin{array}{l}\text { transposition of the metacarpal } \\
\text { bone stump after distraction }\end{array}$ & & & & 13 & 20 & 9 & 3 & & 45 \\
\hline Total (fingers): & $\begin{array}{l}1 \\
(0.5 \%)\end{array}$ & $\begin{array}{l}2 \\
(1.0 \%)\end{array}$ & $\begin{array}{l}4 \\
(1.9 \%)\end{array}$ & $\begin{array}{l}64 \\
(31.5 \%)\end{array}$ & $\begin{array}{l}81 \\
(\mathbf{3 9 . 9 \% )}\end{array}$ & $\begin{array}{l}26 \\
(12.8 \%)\end{array}$ & $\begin{array}{l}9 \\
(4.4 \%)\end{array}$ & $\begin{array}{l}16 \\
(7.9 \%)\end{array}$ & $\begin{array}{l}203 \\
(\mathbf{1 0 0 \%})\end{array}$ \\
\hline
\end{tabular}

*Figures indicate the following levels of amputation: 1 - middle phalanx; 2 - distal third of the proximal phalanx; 3 middle third of the proximal phalanx; 4 - proximal third of the proximal phalanx; 5 - distal third of metacarpal bone; 6 - middle third of metacarpal bone; 7 - proximal third of metacarpal bone; 8 - metacarpal bones.

In the cases of secondary reconstruction, the transposition of segments of fingers I (12 $6.5 \%)$, II (98 - 53.3\%), III (46-25.0\%), IV (26-14.1\%), V (2-1.1\%) was performed, and in the cases of primary reconstruction, the transposition of fingers II (14), III (2), IV (3) and finger stumps was performed. The distal fragment of the first ray stump was transferred only axially for the reconstruction of finger I. The majority of donor segments (deformed fingers, finger and metacarpal bone stumps) had cicatricial alterations of different degrees, depth, and length in soft tissues (166$90.2 \%$ ). This provided or engraved the deformities of segments and disturbances in the functioning and blood supply due to secondary changes of vessels or their damage, which significantly 
increased the risk of ischemic complications. For this reason, the segments were transposed by the method of Hilgenfeld-Shushkov [6] on a permanent typically formed palmar vascular-nervous pedicle that contained a 1.5-2 cm wide skin flap used as an additional source of blood supply and lymphovenous drainage. The prevention of ischemic complications was provided by the maximum preservation and microsurgical reconstruction of the damaged palmar digital arteries, dorsal subcutaneous veins, formation of an additional dorsal feeding cutaneous-vascular or venous pedicle, as well as pre-training (delayed reconstruction) of the segment against ischemia. Such an approach can be used during secondary reconstruction and in patients with cicatricial tissues. It included osteotomy and mobilization of the donor segment on the palmar cutaneous-vascular pedicle with its further fixation to its place in the position of rotation, which creates moderate ischemia because of the vessel pedicle compression controlled with clinical and instrumental methods. In the majority of cases, delayed reconstruction of the segment included its distraction with the Ilizarov frame. This procedure provided dosed ischemic load on tissues and elongation of feeding pedicles (dorsal and palmar) to the required length (author's certificate No. 1775883; patents of the RF Nos. 2069545; 2093092; 2072807; 2152184; 2145812; 2120246; 2391930). The transposition of the preformed segment was performed at the second stage of the surgery in 14-21 days. If the segment was distracted, the second surgery was performed after its completion. Depending on the state of blood circulation of the complex, it was transferred on one (palmar) or two (palmar and dorsal) permanent feeding pedicles. The specified operations were especially feasible in patients with cicatricial alterations in soft tissues, severe segments deformities, and obliteration or damage of vessels when it was difficult or impossible to separate them or apply a precise (microsurgical) technique. Based on experimental, morphological, biophysical (IR imaging, laser Doppler flowmetry, rheovasography), and statistical methods of the study, the authors developed a method of mechanical pre-training of the transferred donor finger to endure ischemia [patent of the RF No. 2566190]. The duration of the pre-training (hypoxic preconditioning) was 4-6 days. Along with this, arterial garrot was placed on the donor finger 4 times a day for 5 days. On Day I, the occlusion lasted for 10 minutes, on Day II - 15 minutes, on Day III - 20 minutes, on Day VI - 25 minutes, from Day V - 30 minutes. The criterion of effectiveness of the pre-training was the difference in the value of the temperature increase in the distal part of the finger within 3 minutes after the garrot removal in comparison with the end of the occlusion between the last and first ischemic load by not less than $1.7^{\circ} \mathrm{C}$ regardless of the initial condition, the duration of the pre-training, and the number of compressions.

RESULTS. The retention of all segments, even severely damaged, was achieved during the primary reconstruction. After the secondary reconstruction, the necrosis of the transferred finger stump (1), partial necrosis of the nail phalanx of the transferred deformed finger (1), partial necrosis 
of soft tissues on the dorsal surface of the finger (1), and the finger stump (2) was observed. In all the cases, ischemic complications of different degrees of severity, primarily, venous complications (34.3\%) were observed during the transposition of cicatricial and deformed segments. In 1 case of complete necrosis $(1-0.49 \%)$, the function of the handgrip was not reconstructed. All the other complications were resolved and did not influence the treatment outcome. Original approaches to the transposition and pre-training against ischemic provided the retention of all the segments.

The effectiveness of the treatment was evaluated by the improved method of Belousov (1984). A very good outcome was registered in $41.6 \%$, good - in $16.9 \%$, satisfactory - in $34.8 \%$, unsatisfactory - in $6.7 \%$ of cases. No unsatisfactory outcomes were registered after the primary transposition. The results of the transposition of the deformed finger, finger stump, and metacarpal bone were not significantly different $(\mathrm{P}>0.05)$. A comparative analysis of the results of the conventional and original methods was performed. The compared groups did not differ significantly by the age $(\mathrm{P}=0.99)$, sex $(\mathrm{P}=0.99)$, etiology of injury $(\mathrm{P}=0.61-0.71)$, and types of hand defects $(\mathrm{P}=0.6-0.8)$. It was revealed that the application of original methods allowed the surgeons to transpose finger stumps in patients with a more proximal level of amputation $(\mathrm{P}=0.02)$. Besides, the distraction of the segment allowed the surgeons to transpose the finger and metacarpal bone stump with more ulnar location ( $\mathrm{P}=0.01$ and $\mathrm{P}=0.0001$, respectively). Two-point discrimination on the reconstructed finger after the transposition was $6.28 \pm 0.77 \mathrm{~mm}$, the metacarpal bone $-7.3 \pm 0.6 \mathrm{~mm}$, and the intact finger $-3.1 \pm 0.3 \mathrm{~mm}$. Unsatisfactory results were explained by the total defect of the first metacarpal bone and not the character, degree of deformity, blood circulation disturbances in the transferred segment, and its initial function disorders. The function of handgrip and the stability of finger bones, confirmed by biochemical, roentgenometric, and morphological tests, were restored in patients with different hand deformities of a mechanical, gunshot, and thermal etiology, including those with vast cicatricial tissues and disturbances of magistrate blood circulation with minimum donor area damage.

The obtained functional results are comparable with the available published data [1], even though the authors significantly expanded the set of indications for this method due to the transposition of pathologically altered segments.

\section{Clinical case.}

Man, 42 years old, was admitted to the clinic with total deformity of the radial side of the right hand; flexing dermato-teno-arthrogenic contractures of fingers II, III and IV; deformity of soft tissues and bones; damage of palmar digital arteries of finger II, extensive contracture of the right hand after severe mechanical injury (Figures 2-4). 


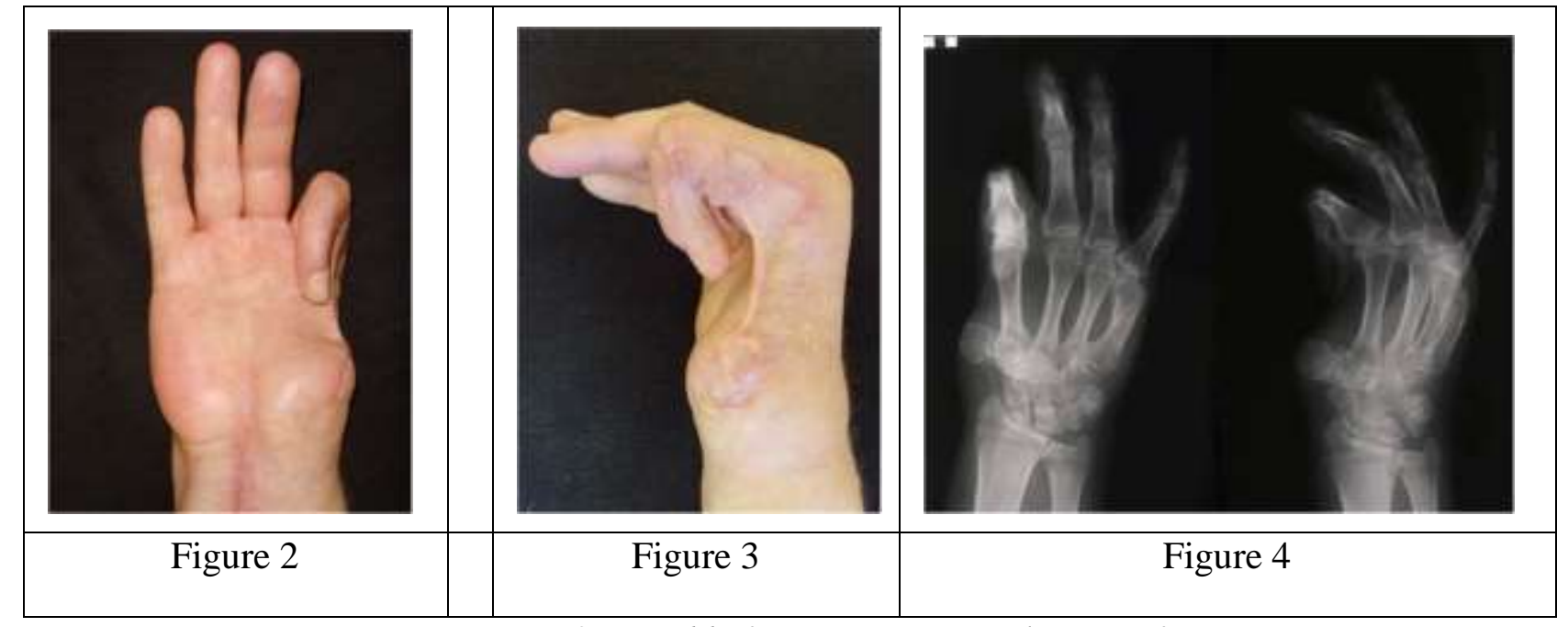

Figure 2. Hand before operation (palmar surface)

Figure 3. Hand before operation (lateral surface)

Figure 4. X-ray of hand before the operation

In 2 years and 5 months after the injury, single-stage transposition of the most deformed finger to the position of finger I was performed. The finger was transferred on the dorsal and plantar feeding pedicles after the skin flap plasty and pre-training against ischemia by the developed method.

The transferred segment and skin flaps completely retained, the wounds healed per primam, which allowed the surgeons to form a thumb and first web space (Figures 5 and 6). The handgrip was reconstructed despite the initial severe deformity and dysfunction of the donor segment (Figure 7).

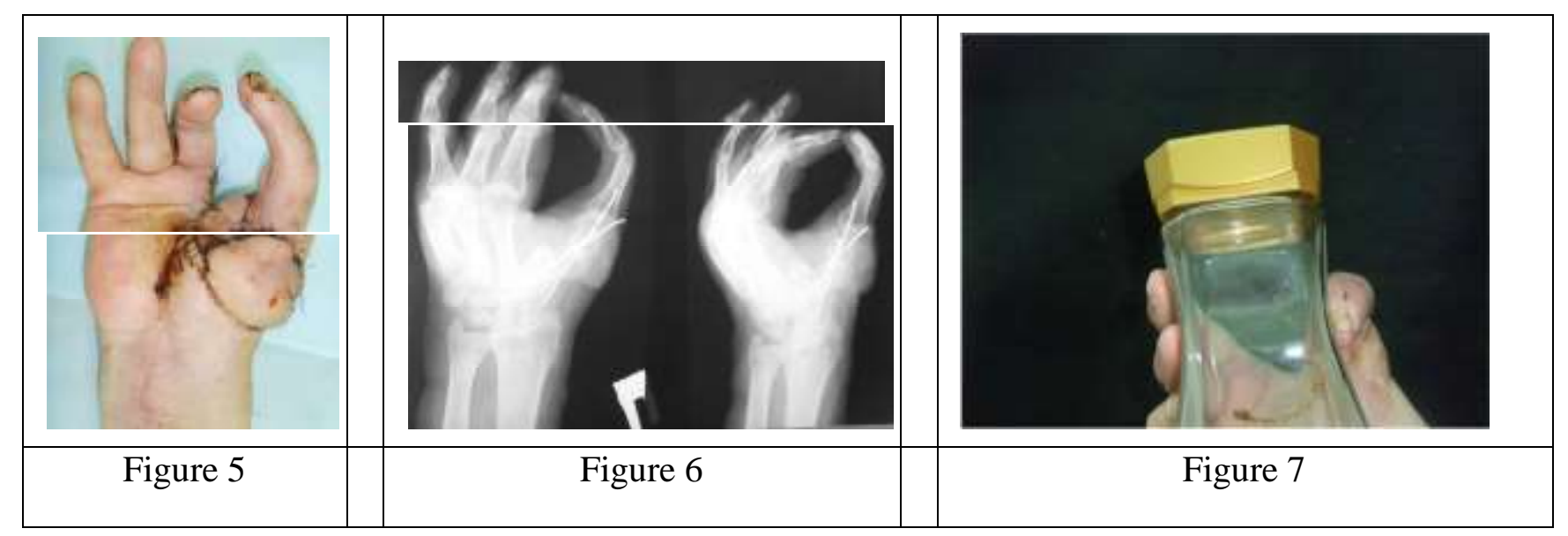

Figure 5. Short-term outcome of the transposition of finger II

Figure 6. X-ray of the hand after the surgery

Figure 7. Handgrip: 1 year after the surgery 
The published data and the authors' experience show that primary or venous revascularization of the donor segment is feasible only in cases when the vessels are not damaged, intact tissues are preserved or there are no severe cicatricial alterations when the risk of ischemic complications is relatively low [7-9]. The results of the present study showed that the hypoxic preconditioning of the segment was an effective method of prevention of post-operational ischemic complications. It activates the mechanisms of adaptation at different levels of systemic organization of tissues in response to a short, mild, and undamaging ischemic stimulus. Dosed controlled ischemic load on tissues, achieved during pre-training of the transferred complex, improves its resistance to hypoxia, induces cellular metabolic adaptation, the longitudinal orientation of pedicle vessels, and an increase in the blood rate, which improves the retention of the segment tissues. Besides, an elongation of feeding pedicles is observed, which allows for the transposition of remote stump of the metacarpal bone and any three-phalanges finger at the primal levels, including the cases with two feeding pedicles, via the remaining fingers or stumps, and perform the reconstruction of the first and/or one of the other fingers with minimal damage to the donor area [10].

The obtained results showed that the developed approaches expand the set of indications for the transposition of hand segments and provide adequate functional results even in cases with severe cicatricial deformities.

CONCLUSIONS. Ischemic preconditioning and preformation of cicatricial donor segments provided their $100 \%$ retention and restoration of the handgrip in the majority of patients $(93.3 \%)$ with different types of hand deformity of a mechanical, gunshot, and thermal etiology.

\section{FINANCIAL SUPPORT AND SPONSORSHIP}

Nil.

\section{CONFLICTS OF INTEREST}

The authors declare no conflict of interest

\section{SUPPLEMENTARY DATA (DOI)}

\section{REFERENCES}

1. Ishida O., Taniguchi Y., Sunagawa T. et al. Pollicization of the index finger for traumatic thumb amputation. Plastic and Reconstructive Surgery, 2006, vol. 117, no. 3, pp. 909-914, doi 10.1097/01.prs.0000200627.74575.15. 
2. Goldfarb C.A., Monroe E., Steffen J. et al. Incidence and treatment of complications suboptimal outcomes and functional deficiencies after pollicization. Journal of Hand Surgery, 2009, vol. 2, no. 34, pp. 1291-1297, doi 10.1016/j.jhsa.2009.04.001.

3. Manske P.R. Index pollicization for thumb deficiency. Tech Hand Up Extrem Surgery, 2010, no. 14, pp. 22-32, doi 10.1097/bth.0b013e3181d44545.

4. Thate M.R., Nehete S., Garude K. et al. Unfavourable results in pollicisation. Indian Journal of Plastic Surgery, 2013, vol. 46, no. 2, pp. 303-311, doi 10.4103/09700358.118609 .

5. Bravo C.J., Horton T., Moran S.L. et al. Traumatized index finger pollicization for thumb reconstruction. Journal of Hand Surgery, 2008, vol. 33, no. 2, pp. 257-262, doi 10.1016/j.jhsa.2007.11.008.

6. Gohritz A., Dellon A.L., Muller F.E. et al. Otto Hilgenfeldt (1900-1983): tribute to an important pioneer of European hand surgery. Journal of Hand Surgery (European Volume), 2012, vol. 37, no. 3, pp. 205-210, doi 10.1177/1753193411421098.

7. Chafik D., Harness N.G., Lawrence J.F. Unusual arterial anatomy in a case of index finger pollicizatio. Journal of Hand Surgery, 2006, vol. 31, no. 2, p. 347, doi 10.1016/j.jhsb.2006.02.001.

8. Kumar B., Acharya A., A.K. Bhat A re-look at pollicization. Indian Journal of Plastic Surgery, 2011, vol. 2, no. 44, pp. 266-275, doi 10.4103/0970-0358.85348.

9. Kozin S.H. Pollicization: The Concept, Technical Details and Outcome. Clinics in Orthopedic Surgery, 2012, vol. 4, no. 1, pp. 18-35, doi 10.4055/cios.2012.4.1.18.

10. Azolov V.V., Alexandrov N.M., Petrov S.V. Application of Ilizarov Device for Transfer of Deformed Digits, Digital Stumps and Metacarpal Stumps. Journal of Hand Surgery, 2009, vol. 34, no. 5, pp. 1531-1540, doi 10.1016/j.jhsa.2009.05.013. 